\title{
Immunotherapy for bladder cancer
}

\author{
This article was published in the following Dove Press journal: \\ Research and Reports in Urology \\ 4 May 2015 \\ Number of times this article has been viewed
}

\section{Oliver Fuge' \\ Nikhil Vasdev' \\ Paula Allchorne ${ }^{2}$ \\ James SA Green ${ }^{2}$ \\ 'Department of Urology, Lister Hospital, Stevenage, UK; ${ }^{2}$ Department of Urology, Bartshealth NHS Trust, Whipps Cross Rd, London, UK}

Correspondence: James SA Green Department of Urology, Barts Health NHS Trust (Whipps Cross), Whipps Cross Rd, London EII INR, UK Email James.Green@bartshealth.nhs.uk
Abstract: It is nearly 40 years since Bacillus Calmette-Guérin (BCG) was first used as an immunotherapy to treat superficial bladder cancer. Despite its limitations, to date it has not been surpassed by any other treatment. As a better understanding of its mechanism of action and the clinical response to it have evolved, some of the questions around optimal dosing and treatment protocols have been answered. However, its potential for toxicity and failure to produce the desired clinical effect in a significant cohort of patients presents an ongoing challenge to clinicians and researchers alike. This review summarizes the evidence behind the established mechanism of action of BCG in bladder cancer, highlighting the extensive array of immune molecules that have been implicated in its action. The clinical aspects of BCG are discussed, including its role in reducing recurrence and progression, the optimal treatment regime, toxicity and, in light of new evidence, whether or not there is a superior BCG strain. The problems of toxicity and non-responders to BCG have led to development of new techniques aimed at addressing these pitfalls. The progress made in the laboratory has led to the identification of novel targets for the development of new immunotherapies. This includes the potential augmentation of BCG with various immune factors through to techniques avoiding the use of BCG altogether; for example, using interferon-activated mononuclear cells, BCG cell wall, or BCG cell wall skeleton. The potential role of gene, virus, or photodynamic therapy as an alternative to BCG is also reviewed. Recent interest in the immune check point system has led to the development of monoclonal antibodies against proteins involved in this pathway. Early findings suggest benefit in metastatic disease, although the role in superficial bladder cancer remains unclear.

Keywords: immunotherapy, Bacillus Calmette-Guerin, bladder cancer

\section{Introduction}

It is coming up to 40 years since the first report of the use of Bacillus Calmette-Guerin (BCG) as an immunotherapy for the treatment of bladder cancer. ${ }^{1}$ Since then, numerous studies have set out to explain how BCG exerts its effect on urothelial tumor cells. Further, several clinical trials have attempted to address the clinical questions relating to BCG immunotherapy, with particular attention paid to dosing regimens, induction and maintenance therapy, comparison with the cytotoxic chemotherapeutic agents, and combination therapy, for example, using interferon (IFN)- $\alpha{ }^{2}$

The anatomical properties of the bladder make it an excellent organ for this type of therapy. Its hollow structure with a ready-made access route via the urethra facilitates direct intravesical administration of antitumor agents which are relatively contained, thus minimizing, although not completely preventing, toxic systemic effects. 
Moreover, the response to these agents can subsequently be monitored with relative ease when compared with other organs through surveillance cystoscopy and biopsy. Non-muscle-invasive bladder cancer (NMIBC) represents around $70 \%$ of the 70,000 new bladder cancer cases diagnosed in the USA each year. ${ }^{3}$ The development of BCG as an immunotherapy for NMIBC and its proven effects of reducing recurrence and progression has revolutionized the treatment of this malignancy. ${ }^{4}$ There are also reports that, at present, BCG is underused, so better awareness of its indications could potentially help a greater number of patients with NMIBC. 5,6 This review summarizes the evidence to date and also looks at future prospects for the use of immunotherapy for bladder cancer.

\section{History of the development of BCG}

BCG was developed as a vaccine against tuberculosis (TB). It is a live attenuated vaccine originally produced from the same bacterium that gives rise to bovine TB, ie, Mycobacterium bovis. Attenuation was achieved through manipulation of the bacillus by serial growths on a culture medium. This resulted in a gradual loss of the genes producing virulence such that it could be safely inoculated into humans. ${ }^{7}$ Importantly, it retains enough similarity to Mycobacterium tuberculosis that when a human is vaccinated with BCG a degree of immunity to TB ensues. It was originally developed by Calmette and Guerin at the beginning of the 20th century using a glycerinated bile potato medium in a process that began in 1908, leading to its first clinical use as a vaccine for TB in $1921 .{ }^{8}$ On average, a 50\% reduced risk of TB with BCG can be expected according to a meta-analysis published in 1994. ${ }^{9}$ However, reductions in TB due to BCG do range considerably, with figures from $0 \%$ to $80 \%$ reported. ${ }^{10}$ It is thought that this, in part, reflects the genetic variability between the different strain preparations created around the world. ${ }^{7,10}$

\section{History of the use of BCG in bladder cancer}

In 1929, Pearl noted that patients with tuberculosis had lower rates of cancer when examined at autopsy. ${ }^{11}$ This observation was arguably the first step in the journey that ultimately led to the treatment of bladder cancer with BCG. Animal studies in the 1950s confirmed the positive effects of BCG on cancer rates but it was not until 1976 when Morales et al published the seminal paper on the use of intravesical BCG in human patients. ${ }^{1}$ Nearly 40 years later, BCG remains an important therapeutic tool in NMIBC.

\section{Mechanism of action}

Many would argue that the exact mechanism of action of BCG in its ability to treat bladder cancer is not fully understood. ${ }^{12}$ However, the profound effect of BCG on stimulation of the immune system is well recognized. ${ }^{12,13}$ What is known for certain is that several immune molecules and cell types are involved ${ }^{12,14,15}$ and, as the body of work on the subject grows, it is apparent that the effects of BCG on the immune system are complex, multifactorial, and likely to evolve as our understanding increases.

Evidence to date supports the idea that the antitumor effects of BCG are produced by an interplay between the direct effects on tumor cells by BCG infection and the host's immune response. ${ }^{15}$ The result of this immune activation is to improve recognition and subsequent destruction of tumor cells through non-specific and specific cell-mediated mechanisms. $^{14}$

One approach to understanding the effects of BCG is to separate the complex reactions into the following three categories (Table 1). This scheme is advocated, by Kawai et al, amongst others. ${ }^{15}$

\section{Infection of urothelial cells}

Ratliff demonstrated the role of fibronectin (a glycoprotein of the extracellular matrix) in the attachment of BCG to tumor cells in the late 1980s. ${ }^{16}$ BCG is then internalized into the tumor cells, a step that appears to be fundamental to the subsequent immune response. This was demonstrated in experiments where an antifibronectin antibody inhibited

Table I Summary of key steps and mediators in the mechanism of action of BCG immunotherapy

\begin{tabular}{|c|c|}
\hline Steps in BCG activity & Mediated by \\
\hline $\begin{array}{l}\text { I. Infection of urothelial } \\
\text { and/or bladder cancer cells }\end{array}$ & Fibronectin \\
\hline $\begin{array}{l}\text { 2. Induction of immune } \\
\text { reaction }\end{array}$ & $\begin{array}{l}\text { Cell types: granulocytes, T-helper cells, } \\
\text { dendritic cells, and macrophages } \\
\text { Immune molecules: MHC class I, CD4+, } \\
\text { various cytokines including IL-I, IL-2, IL-6, } \\
\text { IL-8, IL-I0, IL-I2, IL-I7, TNF- } \alpha \text {, and IFN- } \gamma \text {. }\end{array}$ \\
\hline $\begin{array}{l}\text { 3. Induction of antitumor } \\
\text { effects }\end{array}$ & $\begin{array}{l}\text { ThI cells (acquired immunity) via } \\
\text { CD4+ T-cells and CD8+ cytotoxic } \\
\text { T lymphocytes (driven by IL-2, TNF, } \\
\text { IL-I2, and IFN- } \gamma \text { ) } \\
\text { Th2-cell (innate immunity) through NK } \\
\text { cells (driven by IL-4, IL-5, IL-6, and IL-I0) } \\
\text { Neutrophil recruitment (via IL- I7 release) } \\
\text { and macrophages. }\end{array}$ \\
\hline
\end{tabular}

Abbreviations: IL, interleukin; MHC, major histocompatibility complex; BCG, Bacillus Calmette-Guerin; NK, natural killer; TNF- $\alpha$, tumor necrosis factor alpha; IFN- $\gamma$, interferon gamma. 
the antitumor effect of BCG. ${ }^{17}$ In a mouse model, BCG has been found in urothelial cells within 24 hours of instillation. ${ }^{18}$ This finding has been supported by in vitro studies in human bladder cancer cell lines. ${ }^{19,20}$

\section{Induction of immune reaction}

This infection of urothelial cells by BCG leads to stimulation of the local immune response via activation of the reticuloendothelial system. ${ }^{21}$ Granulocytes, macrophages, and T-helper (Th) cells all appear to facilitate this process. The internalization of BCG, as with other microbes, mediates the antigenprocessing functions of antigen-presenting cells. ${ }^{22} \mathrm{BCG}$ also increases the surface expression of major histocompatibility complex class I in urothelial tumor cells. ${ }^{23}$ It is this enhancement of antigen presentation following BCG that suggests a T-cell/major histocompatibility complex pathway is involved in the antitumor response. In mouse bladder carcinoma cells, presentation of antigen to BCG-specific CD4+ T-cells has been demonstrated. ${ }^{24}$ These findings support the idea that not only the host's circulating antigen-presenting cells but also infected bladder tumor cells are involved in initiating the immune response.

Several cytokines have been implicated in this cellmediated response, including interleukin (IL)-1, IL-2, IL-6, IL-8, IL-10, IL-12, tumor necrosis factor- $\alpha$, and IFN. ${ }^{25}$ This has been demonstrated by analysis of urine following administration of BCG. IL-1, IL-6, and IL-8 are increased after the first BCG instillation. ${ }^{26,27}$ However, with the majority of the other cytokines, an increase is not seen until the second dose of BCG; moreover, the concentrations increase with successive treatments. ${ }^{28,29}$ This biochemical response would appear to explain the clinical response, in immunological terms, obtained after successive BCG instillations. More recently IL-17 has been shown to be involved in the process; release of this cytokine by T-cells in response to BCG appears to result in recruitment of neutrophils to the bladder, which is important for its antitumor effect. ${ }^{30}$

The role of IFN seems to have particular importance. Patard attempted to quantify the local immune response by analyzing the production of IFN in urine following BCG therapy as a means to define the optimal treatment regime. ${ }^{31}$ This was achieved using an enzyme-linked immunosorbent assay test kit. This study revealed that, during a 6-week course of BCG, at least three weekly instillations were required to produce a detectable response in IFN- $\gamma$, which continued to increase with successive treatments. Further, they demonstrated that tumor-associated antigens and major histocompatibility complex molecules were induced on urothelial cells following treatment in five of the seven patients analyzed. The authors proposed that this simple test could be used as a way to rationalize treatment dose and duration.

\section{Antitumor activity}

\section{Acquired versus innate immunity}

The idea that Th1 cell-mediated (or acquired) immunity is responsible for producing the beneficial antitumor activity stimulated by BCG is well documented. ${ }^{14}$ This effect is produced by CD4+ T-cell and CD8+ cytotoxic T lymphocyte activity. However, there is evidence that the innate or humoral response (mediated by the Th2 arm of the immune system) also serves to induce an antitumor effect. Mice deficient in natural killer cells (or when given anti-natural killer antibody) failed to respond to BCG therapy. ${ }^{32}$

\section{Role of neutrophils, macrophages, and dendritic cells}

There is increasing evidence to support the role of neutrophils in BCG immunotherapy for bladder cancer. ${ }^{33}$ Neutrophils are the major white blood cell component present in urine following BCG therapy, the degree of which appears to predict tolerance and outcome of intravesical BCG immunotherapy. ${ }^{34}$ In a study of 72 patients, Saint et al showed that a high leukocyturia count following BCG correlated with a reduction in recurrence; further, a high level also predicted the severity and duration of adverse events. The preliminary results of this study suggest that the degree of leukocyturia might be used as a means by which to adapt BCG maintenance regimes to the individual patient. ${ }^{34}$ However, as in many other areas of BCG immunotherapy research, larger-scale, randomized trials are required before this notion becomes clinically relevant.

Macrophages have also been identified as likely cell types involved in BCG-induced antitumor activity. ${ }^{35,36}$ Macrophages are one of the first-line agents in the body's defense against mycobacterial infection, and their role in BCG-induced cytotoxicity, has arguably been underestimated to date, as evidenced by the relative lack of in vivo studies in this area. ${ }^{12}$ They appear to have a role both in direct tumor cell death and presentation of antigens to cells of the adaptive immune system. ${ }^{35}$

The role of both macrophages and IFN- $\gamma$ in the antitumor response is further supported in a study by Thiounn et al. ${ }^{37}$ Using an in vitro culture of autologous mononuclear cells activated by IFN- $\gamma$ that were introduced back into the bladders of patients with G2 and G3 p Ta disease, this group showed a statistically significant reduction in rate of recurrence. As well as demonstrating the potential role of macrophages activated 
by IFN- $\gamma$ in the pathway of BCG-mediated immunotherapy, this study carries with it the potential benefit of bypassing BCG-related toxic effects by not using the mycobacterium directly. However, these results have yet to be validated in larger-scale studies.

The observation that a patient's prognosis is better if there are increased numbers of urinary dendritic cells after BCG therapy led Naoe et al to investigate whether dendritic cells preactivated by BCG in vitro increase the numbers of natural killer T-cells and $\gamma \delta$ T-cells. ${ }^{36}$ As well as showing this to be the case, the authors also reported cytotoxic activity of the resulting lymphocytes against a bladder cancer cell line, a finding that reveals yet another cell type potentially implicated in the mechanism of BCG-mediated antitumor activity.

\section{Summary of BCG action}

The immune response to BCG can be summarized as follows: infection of urothelial and bladder tumor cells by BCG results in internalization of BCG, which increases the expression of antigen-presenting molecules. This induces an immune response via cytokine release. Th1 cytokines (IL-2, tumor necrosis factor, IL-12, and IFN- $\gamma$ ) and Th2 cytokines (IL-4, IL-5, IL-6, and IL-10) along with IL-8 and IL-17 are all implicated. This complex immune cascade induces antitumor activity mediated by cytotoxic $\mathrm{T}$ lymphocytes, natural killer cells, neutrophils, and macrophages.

\section{Can response to therapy be predicted?}

The potential for concentrations of urinary neutrophils to predict antitumor activity has already been discussed; however, other parts of the immune response have also been put forward to perform the same purpose. Measurement of tumor-infiltrating dendritic cells and tumor-associated macrophages has been proposed as a means by which to predict the response to BCG therapy. Ayari et al assessed levels of CD83+ dendritic cells and CD68+ macrophages in patients with high-risk NMIBC treated with transurethral resection (TUR) of bladder tumor (TURBT) and maintenance BCG. ${ }^{38}$ Their results suggested that patients with low levels of CD83+ tumor-infiltrating dendritic cells responded better to maintenance BCG than patients with higher levels at the time of resection. In the same cohort, a similar effect was seen in those patients who recruited more CD68+ tumor-associated macrophages, ie, there was an increased risk of recurrence. Further studies are required to confirm these preliminary findings, but if validated, the pretreatment levels of these immune cells may have relevance in determining the treatment regime for individual patients.

BCG is known to induce tumor necrosis factor-related apoptosis-inducing ligand (TRAIL) via release of IFN. ${ }^{13}$ BCG appears to be responsible for upregulation of TRAIL, as shown in a study that compared baseline and subsequent urinary TRAIL levels in patients given intravesical BCG. ${ }^{39}$ This upregulation predicted the response to BCG therapy, with those responding (defined as more than 12 months tumor-free) expressing levels of urinary TRAIL approximately three times that of those who did not. The same study demonstrated the in vitro death of bladder cells by TRAIL, highlighting the probable importance of this molecule in the action of BCG in bladder cancer.

\section{Clinical perspectives Evidence for reduction of recurrence of bladder cancer by BCG}

There is now a large body of evidence demonstrating the superior efficacy of BCG in the treatment of bladder cancer when compared with TUR alone or TUR and chemotherapy. The European Association of Urology (EAU) guidelines on NMIBC in 2013 report a total of five meta-analyses to substantiate this claim. ${ }^{40}$ In addition, there have been individual randomized controlled trials comparing BCG with epirubicin and IFN, ${ }^{41}$ mitomycin $\mathrm{C},{ }^{42}$ and epirubicin alone, ${ }^{43}$ all of which showed BCG to be the best agent with respect to preventing recurrence.

\section{Role in carcinoma in situ}

Prior to the development of BCG immunotherapy, 52\% of patients with carcinoma in situ (CIS) would go on to develop muscle-invasive disease. The beneficial role of BCG in CIS of the bladder was demonstrated in a study showing a $71 \%$ complete response, again using a 6-week instillation course. ${ }^{44}$ A large, randomized controlled trial by the South West Oncology Group comparing induction plus a 3-week maintenance course versus induction alone using intravesical BCG in CIS showed a clear advantage of the former regimen. ${ }^{45}$ The addition of a 3-week maintenance course beginning at 3 months produced a complete response rate of $84 \%$ and, with ongoing 3-monthly installations, over $70 \%$ of those who responded remained disease-free for over 5 years.

\section{Evidence for prevention of progression by BCG}

As the ability of BCG to prevent tumor recurrence became increasingly well documented, it became less clear how this 
translated into preventing disease progression or attaching mortality. Several studies have attempted to define whether or not BCG immunotherapy can prevent progression. A meta-analysis by Sylvester et al in 2002 identified a total of 24 trials encompassing 4,863 patients comparing TUR and BCG with either TUR alone or TUR with another non-BCG treatment. ${ }^{4}$ This study found a reduction of $27 \%$ in the odds of progression in patients treated with BCG if given any period of maintenance therapy. This finding was supported in a meta-analysis by Böhle and Bock in $2004 .{ }^{46}$ Their study looked at the outcomes from nine clinical trials including a total of 1,277 patients comparing BCG and mitomycin C. Overall, there was no statistically significant difference in the odds ratio for progression between the two groups; however, as determined by Sylvester et al, there was a statistically significant reduction in tumor progression in the subgroup of patients treated with BCG where maintenance therapy was also given. ${ }^{4}$

The 3-year treatment protocol used in the USA by the South West Oncology Group ${ }^{45}$ has been validated in subsequent randomized controlled trials. Sylvester et al put BCG head-to-head with intravesical epirubicin chemotherapy in a large study of patients with intermediate-to-high risk bladder cancer. ${ }^{47}$ This maintenance regimen confirmed reduced recurrence, but for the first time, given the long-term followup in the study design, also showed a benefit with regard to disease-specific survival.

\section{Optimal treatment regime}

Herr and Morales understood that at least 3 weeks of treatment were required in order to induce the delayed hypersensitivity reaction necessary for an immune-mediated effect from therapy. ${ }^{48}$ The supplier of the BCG vaccine used in their study marketed its product in packs containing six $120 \mathrm{mg}$ vials, and this, combined with the knowledge that side effects resolved after a week, led these authors to create a 6-weekly regimen that was used in the original paper. ${ }^{1}$ The effect of this was that tumor recurrence was reduced 12-fold, although this was seen in a small patient group. Further work was required to validate these promising preliminary findings.

The same regimen was employed in the initial controlled clinical trials of BCG in human bladder cancer organized by the National Cancer Institute. Results from these trials were published by two separate groups in 1980 and 1985, respectively, ${ }^{49,50}$ and showed a significant reduction in tumor recurrence.

Ongoing follow-up of the original study revealed that the benefits of BCG (in terms of preventing recurrence, progression, and mortality) are lost after 15 years, and this ultimately led to the development of the concept of maintenance therapy. Extravesical mortality rates of $32 \%$ and $44 \%$, with disease in the ureters and prostatic urethra, respectively, were seen in this cohort of patients. ${ }^{51}$

Lamm showed that compared with a single induction course, the introduction of maintenance BCG reduced tumor recurrence in high-risk patients from $52 \%$ to $25 \%{ }^{45}$ This benefit was seen over a 7-year period.

For maximal efficacy, the latest EAU guidelines recommend giving BCG in a maintenance schedule. ${ }^{40}$ These guidelines highlight the fact that several schedules have been adopted (from 10 instillations over 18 weeks, to 27 over 3 years) but the meta-analysis by Sylvester et al in 2002 failed to identify the most effective regime. ${ }^{4}$

Conclusive evidence for the optimal number of induction instillations and frequency and duration of maintenance therapy is lacking, ${ }^{52}$ and due to the infinite number of possible combinations, is unlikely ever to be realized. Therefore, it is important to use the evidence that is available and weigh this against other factors, such as cost, adverse effects, and patient choice, when deciding upon a regime for an individual patient.

The European Organization for Research and Treatment of Cancer (EORTC) conducted a randomized controlled trial and demonstrated that 3 years of maintenance rather than 1 year was required in order to reduce the recurrence rate in high-risk patients, ${ }^{53}$ the same effect was not observed in intermediate-risk patients, and there was no difference in progression or survival. For this reason, the EAU recommend that BCG immunotherapy be reserved for high-risk disease, and that outside of this category intravesical chemotherapy should be considered as first-line therapy.

To this day, one of the main ongoing challenges regarding BCG maintenance therapy is to establish the most effective treatment regimen. One study of patients receiving BCG for CIS and high-grade bladder tumors compared an induction course with a 6-week maintenance course every 6 months for 2 years, but this protocol showed no benefit over induction alone. ${ }^{54}$ Further studies were therefore required to determine if there was a more effective maintenance protocol.

As the shelf-life of BCG-induced immune stimulation is far exceeded by the lifelong risk of tumor recurrence and progression, maintenance therapy is a vital part of BCG therapy for bladder cancer. In a randomized controlled trial, Lamm showed that BCG given in 3-weekly cycles at intervals of 3,6 , and then 6 months up to 3 years not only reduced recurrence, but also worsening of disease, defined as stage progression or need for surgery, radiotherapy or 
systemic chemotherapy. ${ }^{45}$ The positive effect of maintenance was backed up in a meta-analysis comparing induction plus maintenance BCG with induction alone, which demonstrated that addition of a maintenance regime achieves a reduction in tumor progression of $37 \% .{ }^{4}$ This study also assessed cancerspecific mortality, as well as progression, comparing BCG with surgery and chemotherapy. Although a 19\% reduction in mortality with BCG was shown, this was not statistically significant.

There are some data supporting the need to continue maintenance for a period of at least 3 years, as evidenced by a study comparing a 1 -year with a 3 -year maintenance regime for high-risk disease only. ${ }^{55}$ At present there are no studies looking beyond a 3-year time frame, but it is reasonable to assume that, as the effects of BCG wane with time, there is a need to provide ongoing BCG maintenance therapy in view of the fact that the risk of recurrence and progression remains with the patient lifelong. Clearly, further studies in this area are required in order to provide the optimal evidence for best clinical practice.

Some groups firmly advocate the benefits of 3 years over 1 year of maintenance therapy, especially in relation to CIS, ${ }^{45}$ whereas EAU guidelines support the use of any maintenance therapy exceeding 1 year based primarily on the meta-analysis by Sylvester et al. ${ }^{4}$ An alternative method, supported by Herr et al and described in 2011, rejects maintenance therapy altogether and adopts a more pragmatic patient-based approach and favors selective retreatment. ${ }^{56}$ In a large cohort of 1,021 high-risk patients, a 6-week induction course of BCG was given. Patients who responded did not receive any maintenance therapy but were retreated with BCG when relapse occurred. The results were compared with those of published randomized maintenance trials after a minimum follow-up of 5 years using tumor and progression-free survival rates as end points. The authors concluded that their results compared favorably, with recurrence-free and progression-free survival rates of $46 \%$ and $89 \%$, respectively. Although recurrence rates were somewhat higher than in previously published studies, progression rates were similar. Overall, $32 \%$ required a further course of BCG. Put another way, approximately seven in ten patients who would otherwise have received maintenance BCG (in accordance with the generally accepted view of optimal therapy) did not need it with this approach. Clearly, this could have significant implications for both individual patients and health care providers alike, as well as reducing the demand on periodically threatened worldwide supplies of BCG. However, at present, the overwhelming body of evidence does not support this technique, but it does raise some interesting questions and may provide solutions to some of the problems surrounding BCG therapy.

\section{Optimal dose}

In order to reduce the potential side effects of BCG, studies have explored the impact of a reduced dose on both efficacy and toxicity. ${ }^{53,55,57}$ The effect of this approach remains controversial and further assessment is arguably warranted. One-third standard dose (but not one-sixth) has been shown to be adequate in intermediate-risk cancers with respect to efficacy. ${ }^{58}$ There appears to be no difference between severe (ie, systemic) toxicity between normal and reduced dose groups, although there is less incidence of local side effects with a reduced dose.$^{59} \mathrm{An}$ EORTC trial failed to establish any difference in toxicity between standard and one-third dose protocols..$^{53}$ This randomized controlled trial included four arms, ie, one-third dose and full dose BCG for 1 and 3 years each. Moreover, the one-third dose was associated with a higher disease recurrence rate, which was even more evident when given for just 1 year of maintenance therapy. There was no difference between rates of progression or death between the study groups. However, this trial did show that an additional 2 years of treatment with full-dose BCG conferred no benefit for intermediate-risk patients.

A more recent EORTC publication paid particular attention to the side effects in the same study design..$^{55} \mathrm{~A}$ large proportion $(62.8 \%)$ of the 1,316 patients reported local side effects, with no statistically significant difference between the four groups. The previously reported systemic BCGrelated side effects in the region of 5\% were vastly exceeded in this study, at a level of $30.6 \%$. There was also a notable dropout rate of $7.8 \%$, which tended to occur more often in the 1 st year of treatment, and we know from the meta-analysis by Sylvester et al that this period is the minimum critical time maintenance therapy should exceed.

In contrast, The Cancer Committee of the French Urological Association recently published a randomized controlled trial comparing one-third full dose with fewer instillations. ${ }^{57}$ It compared three weekly instillations every 3 months with every 6 months for 3 years and found no statistically significant difference in either disease recurrence or progression. This study also confirmed the findings of earlier studies, in that no difference in toxicity was evident in the group receiving less BCG overall. However, this study is limited by its relatively short follow-up period of just 2 years.

In conclusion, the majority of the studies published to date tell us that reducing the BCG dose seems to have little effect 
on minimizing side effects and systemic toxicity, and may be detrimental to the beneficial effects of BCG immunotherapy for bladder cancer, particularly in high-risk patients.

\section{Toxicity of BCG}

Generally speaking, BCG is fairly well tolerated, but can be fatal (albeit rarely), which must be considered a serious limitation to its clinical use. ${ }^{60}$ Fatalities from disseminated BCG tend to occur in immunocompromised patients, so systemic conditions or pharmacotherapies that interfere with the immune system must be considered prior to initiating therapy. More commonly, BCG produces irritating lower urinary tract symptoms, including frequency and dysuria, which tend to resolve within a few days. There is limited evidence in support of the fact that the presence of BCG-related symptoms equates to a better outcome, ${ }^{61}$ and these adverse effects might act as a surrogate marker of the underlying immunological response. This is worth considering when planning the frequency of follow-up surveillance cystoscopy and also when counseling patients with side effects (ie, they might be more inclined to tolerate these if they know the treatment is having a beneficial effect). Equally, it may be that this subset of symptomatic patients (with a more profound immunological response) needs less frequent maintenance courses, but further research is required to clarify these ideas. Of note, when compared specifically with fever, no relationship with efficacy was identified in the EORTC data, which somewhat argues against a relationship between toxicity and efficacy. ${ }^{62}$

Less common manifestations of BCG toxicity based on an analysis of 2,602 patients undergoing intravesical treatment are summarized in Table 2, and represent less than 5\% of cases. ${ }^{63}$

Severe reactions to $\mathrm{BCG}$ are due to intravenous absorption of the organism and often follow traumatic catheterization. ${ }^{63}$

Table 2 Incidence of non-cystitis Bacillus Calmette-Guerinrelated toxicity

\begin{tabular}{ll}
\hline Side effect & Incidence (\%) \\
\hline Fever $\left(>39^{\circ} \mathrm{C}\right)$ & 2.9 \\
Significant hematuria & $\mathrm{I}$ \\
Prostatitis & 0.9 \\
Systemic infection with pulmonary & 0.7 \\
and/or hepatic involvement & \\
Arthralgia & 0.5 \\
Epididymitis & 0.4 \\
Sepsis (life-threatening) & 0.4 \\
Rash & 0.3 \\
Renal abscess & 0.2 \\
\hline
\end{tabular}

Concurrent cystitis is also believed to contribute to severe reactions. ${ }^{60}$ The advice regarding absolute contraindications to BCG in the EAU guidelines reflects these findings (Table 3). ${ }^{40}$ However, it is recommended that in cases of sterile pyuria or asymptomatic bacteriuria, BCG can be administered and antibiotics are not required. Relative contraindications include immunosuppression and infection with human immunodeficiency virus. However, the evidence for this is not robust, with some studies suggesting no difference in efficacy or safety when compared with patients with an intact immune system. ${ }^{64,65}$

Although the EAU guidelines do not support the use of antibiotics, studies have addressed the idea of giving prophylactic antibiotics with intravesical BCG, and it is probably fair to say the jury is still out with regard to this approach. A randomized, double-blind, prospective, placebo-controlled trial looked at the effect of ofloxacin on BCG-related side effects. ${ }^{66}$ It showed an $18.5 \%$ decrease in moderate and severe adverse effects, which, importantly, translated into improved compliance with BCG treatment. Recurrence (but not progression) rates were also lower in the group given antibiotic prophylaxis; however, the time given to follow-up was only 12 months, so further studies are needed to validate these claims. However, a similarly beneficial effect was not observed in a study in which isoniazid, an anti-TB antibiotic, was given as prophylaxis. ${ }^{67}$

\section{Local side effects}

In accordance with the International Bladder Cancer Group ${ }^{68}$ and EAU advice, ${ }^{40}$ common side effects, including cystitis and/or hematuria, should be treated as follows. For hematuria in the presence of other symptoms, urine needs to be cultured to rule out infection. Persistent hematuria requires direct visualization of the bladder to assess for recurrence of tumor. The advice from the International Bladder Cancer Group for cystitis symptoms is rather more complex. In the first instance, symptoms can be treated with oral non-steroidal anti-inflammatory drugs, which will often result in prompt resolution and instillations can be continued as planned.

Table 3 Absolute contraindications to BCG as per European Association of Urology guidelines

Absolute contraindications to intravesical BCG

- Within 2 weeks of transurethral resection

- Macroscopic hematuria

- Traumatic catheterization

- Symptomatic urinary tract infection

Abbreviation: BCG, Bacillus Calmette-Guerin. 
If symptoms are persistent or worsening, then instillations should be stopped, a urine culture performed, and empirical antibiotics commenced. When empirical antibiotics fail, the advice is to treat according to sensitivities if culture-positive, and if culture-negative, antimicrobial therapy should be changed to a fluoroquinolone for 5 days, to be repeated as required. Intravesical anti-inflammatory preparations should also be considered at this stage. When these measures fail, anti-TB drugs and steroids should be employed. Finally, if there is no improvement in pain, frequency, or urgency, then clearly further BCG is not an option, and radical cystectomy is indicated to address both oncological and symptomatic problems (BCG can cause marked bladder contraction and thus debilitating symptoms for the patient in the absence of an infective cause for symptoms).

\section{Is there a better BCG strain?}

The EAU guidelines on NMIBC (2013 update) report no conclusive evidence for a difference in clinical efficacy between the various BCG strains. ${ }^{40}$ However, in light of new evidence, this statement is now controversial. A prospective randomized controlled trial by Rentsch et al set out to establish if there was a difference in treatment outcomes between two commonly used strains of BCG, ie, Connaught and Tice. ${ }^{69}$ Patients with high-risk NMIBC were given six installations of either BCG Connaught or BCG Tice, and the clinical data were backed up with further tests in murine models. The authors reported a statistically significant benefit of BCG Connaught over BCG Tice with respect to 5-year recurrence-free survival. There was no difference in BCG-related adverse effects. Analysis of the experimental data suggested a more pronounced Th1 response and greater T-cell recruitment in the bladders of BCG Connaught mice. This study suggests that there may be a benefit of BCG Connaught, although further, larger-scale studies will be required in order to validate this finding. Further, if the genes responsible for producing this apparent difference can be confirmed, this could provide valuable information with regard to identification of new targets for potential future immunotherapies.

\section{Future goals and potential new targets in immunotherapy for bladder cancer}

To date, BCG has not been surpassed by any other drug in terms of its ability to reduce disease recurrence and progression, but its potential for side effects and serious toxicity is undoubtedly a limitation to its use. Further, there is still a substantial group of patients, in the region of $30 \%$, who do not respond favorably to BCG and go on to develop muscleinvasive disease.

As a greater understanding of the immunology involved develops, new targets for future drug therapies have been identified. Ultimately, the ideal candidate would be one that produces equivalent or improved, and possibly longerlasting, antitumor effects, is easily manufactured, is better tolerated by patients, and avoids the risk of potentially fatal toxicity. Some of the alternatives to BCG currently being explored are now described.

\section{BCG-cell wall and BCG-cell wall skeleton}

One candidate that has been proposed to replace live $\mathrm{BCG}$ is the BCG cell wall skeleton (BCG-CWS). BCG-CWS is a component of the BCG cell wall (BCG-CW) and can be produced reasonably reliably. For a variety of reasons, including poor solubility and a strong negative charge, the use of BCG-CWS as an immunotherapy in its raw state would not be achievable. However, a Japanese group have recently proposed a novel method of delivering this molecule through packaging it into uniformly sized lipid particles, in a process called the liposome evaporated via emulsified lipid method, in order to improve its bioavailability. ${ }^{70}$ This BCG-CWS nanoparticle has been provisionally tested in rodent models for its antitumor effects. Specifically, it was shown to inhibit tumor growth in mouse bladder tumor (MBT-2) mice and also in a rat model using intravesical administration. Additionally, the same group showed a positive effect on Th1 differentiation in human T-cells.

A similar method to transport BCG-CW into bladder cancer cells has been described in a mouse model using R8-liposomes. ${ }^{71}$ The R8-liposome is akin to an envelope-type virus with the $\mathrm{R} 8$ component being a peptide capable of cell penetration. This has been shown to be effective in vivo in MBT-2 tumors and also in vitro in human bladder cancer cell lines. ${ }^{72}$ Further evaluation in clinical trials is required, but the prospect of using a molecule that induces the same immunological response without the potential for infection is promising.

\section{Toll-like receptors and agonists}

Toll-like receptors (TLRs) are expressed in urothelial cells and are believed to play a role in activation of both the innate and adaptive immune response. These targets have already been utilized in the treatment of other malignancies, for example, in basal cell carcinoma using the drug imiquimod. Variation in TLR expression has been shown, and there is evidence that there are reduced levels in bladder tumors. ${ }^{73}$ 
The same study reported that particular subtypes of the TLR are involved in the response of tumor cells to BCG. Specifically TLR2, TLR4, and TLR9 all seem to be implicated in preventing recurrence with BCG treatment. Agonists of the aforementioned receptors, as well as TLR7, have been created and tested for their antitumor effects, with encouraging results in animal models. ${ }^{73}$ To date, only TLR7 and TLR9 agonists have been tested in clinical trials. ${ }^{74,75}$ Further studies are needed to establish the therapeutic significance of TLR agonists in bladder cancer.

\section{Gene therapy}

Given that the Th1 immune reaction predominates and seems to predict response to $\mathrm{BCG}$, there has been a movement by some research groups to augment the action of BCG by stimulating this arm of the adaptive immune system by modification of standard intravesical therapy. One approach is to administer Th1-stimulating cytokines with BCG, eg, IFN- $\alpha .^{76}$ Although initial data have shown this method to be technically feasible, significant quantities of cytokines are required and, in practical terms, this appears to limit cytokines therapeutic use.

An alternative approach is to employ a recombinant form of BCG, ie, one able to secrete its own array of Th1stimulating cytokines. ${ }^{76}$ So far, several recombinant BCG strains have been created. The hope is that through genetic modification, recombinant BCG might serve to reduce further the rates of disease recurrence and progression, and the initial data seem promising, albeit limited.

\section{Virus therapy}

Activating the antitumor immune response with viruses rather than BCG has recently been proposed as a novel way of treating bladder cancer. At present, this idea is very much in the early stages of development; however, there have been a few preliminary studies suggesting that this may be a useful and productive approach. ${ }^{77}$ Viruses carry the additional benefit of not only inducing an immune response in their own right, but could also act as a vector for delivery of potentially useful genes to stimulate the immune response, thereby augmenting the actions of BCG and possibly avoiding the need to administer BCG altogether.

The first trial to implement a virus-based therapy for bladder cancer used the vaccinia virus. ${ }^{78}$ This was given to patients with known muscle-invasive disease who had a decision to undergo cystectomy already in place. This method permitted a thorough histological examination of the bladder following virus instillation. This study involved just four patients, but no serious adverse effects were noted. Moreover, analysis of specimens revealed virus infection in normal and tumor cells, and an inflammatory response in the form of dendritic cell migration was observed.

A group from the University of Texas reported use of an adenovirus-mediated p53 gene transfer technique for patients with locally advanced bladder cancer. ${ }^{79}$ As in the first study using virus therapy, the patient group was small, but the authors reported the method to be safe and technically feasible. Further, biological activity was noted following intravesical administration of multiple doses of virus particles, with one of the patients seemingly having an antitumor response. A study using a replication-competent granulocytemacrophage colony-stimulating factor-armed adenovirus in patients with superficial bladder cancer who have previously been treated with BCG has shown an overall response rate of $46 \%,{ }^{80}$ which increased to $63.6 \%$ in a multidose cohort. The adverse effects of virus therapy are similar to those seen in BCG immunotherapy, with dysuria, bladder pain and frequency, and systemic influenza-like symptoms all reported. These studies have also highlighted the need to pretreat the urothelium with a GAG solvent in order to improve infection of the virus into the tumor cells.

\section{Role of PDT in BCG-refractory bladder cancer}

\section{Mechanism of action}

Photodynamic therapy (PDT) is not an immune therapy, however, there are some similarities to BCG at the molecular and cellular level. The cytotoxic effect of PDT requires three components to act in unison for the desired effect, ie, a photosensitive molecule, light of a specific wavelength, and oxygen. ${ }^{81,82}$ Further, under these conditions there is a preferential effect on tumor cells, because the photosensitizing drug exhibits an apparent affinity for malignant cells rather than normal tissue. Another possibility to explain this observation is that once taken up by a malignant cell, the drug is retained for much longer than it is by normal cells. ${ }^{83}$ The combination of these three key elements required for PDT to exert its effect results in generation of free radicals, and this results in direct cell death by apoptosis. However, there is also evidence that the immune response generated as a result also plays an important role. The acute inflammatory response secondary to PDT produces infiltration of neutrophils, not dissimilar to that seen post BCG immunotherapy. ${ }^{82}$ It is proposed that tumor-derived antigen presentation is then increased via a T-cell-mediated antitumor effect, in exactly the same way as BCG is now understood to exert its effect. 


\section{Clinical research in PDT for bladder cancer}

There were several preliminary studies using PDT in bladder cancer in the 1990s; however, since then, support for the technique has waned. However, with the emergence of new photosensitizing drugs, interest in PDT has recently been rekindled. In 1993, Canada was the first country to approve a drug for PDT in the treatment of bladder cancer. This led to a trial using Photofrin ${ }^{\circledR}$ in high-risk patients following TURBT ${ }^{84}$ The initial results were encouraging, with a rate of recurrence of $81 \%$ in the control group compared with $39 \%$ in the treatment arm; however, long-term adverse effects have limited its use, with photosensitivity occurring in around a third of patients and over nine in ten experiencing unpleasant urinary symptoms.

These side effects have been reported to be both severe and long-lasting. However, it has been proposed that lower doses can be implemented with meaningful clinical impact and a more favorable side effect profile. Nseyo et al reported a good outcome using three PDT treatments at a reduced dose 6 months apart; although their group of patients was small, 12 of 14 had a complete response. ${ }^{85}$

Uchibayashi et al reported promising outcomes of PDT in patients with BCG-refractory CIS using hematoporphyrin. ${ }^{83}$ Although their study only included 34 patients, the potential longer-term effects of PDT were demonstrated with a median follow-up of over 4 years. A complete response was achieved in $73.5 \%$ of patients. However, disease recurrence was common, occurring in more than half of the patients at 1 year and in over three quarters at 2 years, although these were mainly superficial tumors amenable to TURBT. This study supports the notion that PDT might have a role in bladder-preserving treatments for those who have failed BCG immunotherapy. Again, adverse side effects were not insignificant, with hematuria, frequency, photosensitivity, and a decrease in bladder capacity to around $150-200 \mathrm{~mL}$ all observed.

In 2003, Berger et al assessed the effect of PDT using 5 -aminolevulinic acid in patients with recurrent NMIBC and previously treated with TURBT and/or BCG ${ }^{86}$ This involved intravesical administration (rather than systemic, as in previous studies) of a photosensitizing drug. This group reported that 16 of 31 patients were recurrence-free at 23.7 months. Of those who developed recurrence, the mean time taken to do so was 8.3 months. Dysuria and hematuria were reported adverse effects, but the main benefit of this method seems to have been that skin photosensitivity was avoided. Similar findings were reported in an earlier study by Kriegmair et al. ${ }^{87}$ These studies also add weight to the notion that PDT has the potential to benefit patients in whom BCG has failed and cystectomy is undesirable.

A paper in 2013 reported the efficacy and safety of Radachlorin $^{\circledR}$, a new-generation photosensitive drug, in a group of patients with high-grade NMIBC, again who had failed BCG. ${ }^{88}$ This protocol involved intravenous delivery of the agent, but produced recurrence-free rates of $91 \%$ and $64 \%$ at 12 and 24 months, respectively. The authors reported that there were no severe adverse effects.

A Phase I study, also reported in 2013, assessed the impact of PDT with hexaminolevulinate as an adjuvant treatment in 17 patients after TURBT, given 6 weeks apart on three separate occasions using an intravesical protocol. ${ }^{89}$ The recurrence rates were $47 \%, 76 \%$, and $88 \%$ at 6,9 , and 21 months, respectively. Bladder irritability was reported in 15 of the 17 patients, but this was transient and a cumulative effect with subsequent treatments was not observed. The small number of patients in this study does not allow for definitive conclusions to be drawn, but given the rapid tailoff effect after 6 months, an ongoing maintenance protocol might be required, as with BCG immunotherapy, in order to achieve a more sustained effect in reducing recurrence.

\section{Future goals for PDT}

The main goal for the successful application of PDT in the treatment of bladder cancer seems to be to establish the optimal illumination protocol in conjunction with the appropriate choice of photosensitizing agent and dosing to produce the desired effect whilst minimizing the significant side effect profile. Several new drugs have been identified in recent years, and these appear to have less detrimental effects than the first-generation drugs. ${ }^{90}$

The important benefit of PDT seems to lie in the fact that BCG-refractory patients appear to demonstrate efficacy, thus either delaying or avoiding the need for cystectomy. PDT seems therefore to have emerged as a potential second-line treatment in this substantial group of patients. Further, PDT might have a role in an adjuvant setting for patients undergoing BCG. Murine studies suggest that PDT can augment the antitumor action of BCG immunotherapy ${ }^{91,92}$ but this theory will require validation in human cells and subjects. There also seems to be a role for PDT in other forms of urological malignancy, eg, prostate cancer, and clinical studies assessing its efficacy and safety in this area are in progress (see reviews Pinthus et al, Bozzini et al, and Korbelik et al ${ }^{81,90,91}$ ).

Although the efficacy of PDT has been reported, the majority of studies to date have included small numbers of patients, so larger-scale, randomized controlled trials are 
needed to validate these data. Also, the studies thus far have tended to focus on the effect of PDT on recurrence rates, so future studies will be required to establish the impact on progression..$^{93}$

\section{Immunotherapy in metastatic bladder cancer}

Programmed death-ligand 1 (PD-L1) is expressed on bladder cancer cells, particularly at the invasive and metastatic ends of the disease spectrum. ${ }^{73}$ The role of this molecule, also referred to as B7-H1, appears to be to block the ability of the immune system to recognize and destroy malignant cells. ${ }^{94}$ It is understood that PD-L1 binds to PD-1 and B7.1, which are found on T-cells, rendering them ineffective. ${ }^{95}$ Therefore, by blocking this action, the ability of the immune system to fight tumor cells is restored. It is hoped that identification of inhibitors of this check point system will be of therapeutic benefit, not only in bladder cancer but also in other malignancies, and there is currently a great deal of interest in this area.

In a preliminary Phase I study using MPDL3280A, a monoclonal antibody that targets PD-L1, this theory was tested in 31 patients with metastatic bladder cancer. ${ }^{95}$ The initial results are promising, with a $50 \%$ response rate. Moreover, the treatment was well tolerated, with side effects limited to pyrexia, anemia, anorexia, fatigue, and nausea, and no immune-related adverse effects.

To date, there are no studies assessing the role of anti-PD-L1 drugs in superficial bladder cancer. However, given that PD-L1 appears to be expressed with increasing frequency according to advancing tumor stage and grade, the role of pharmacological blockade in superficial disease might be limited. However, it is plausible that inhibitors of this molecular target might play a role in certain superficial subtypes, perhaps in high-risk NMIBC, and this would have particular relevance in patients who have failed $\mathrm{BCG}$, in whom cystectomy is presently the only effective treatment.

A recent experimental study has assessed the effect of combining BCG with a monoclonal antibody to IL-10 (antiIL-10R1) in a mouse model of bladder cancer. ${ }^{96}$ As previously discussed, the efficacy of BCG is mediated by a Th1 response. IL-10 is known to inhibit the Th1 response and is therefore associated with BCG failure. The study design compared three treatment groups, ie, mice treated with saline, mice treated with BCG alone, and mice treated with BCG and the anti-IL-10R1 monoclonal antibody. Using the anti-IL-10R1 monoclonal antibody with BCG, the authors demonstrated a tumor regression rate of $22 \%$ compared with $6 \%$ in the group treated with BCG alone. Moreover, none of the mice in the combination therapy group developed lung metastases, whereas $36 \%$ and $53 \%$ of mice developed lung metastases in the saline and BCG-alone groups, respectively.

The significance of this study should not be underestimated, and its findings suggest that the anti-IL-10R1 monoclonal antibody in conjunction with BCG confers both a systemic and local antitumor effect, which could have implications for both urologists and oncologists. This interesting study might provide a platform for further research into the use of monoclonal antibodies directed at the immune system in patients with bladder cancer, to support the promising work already commenced in the example of PD-L1 discussed above.

\section{Discussion}

The beneficial effect of intravesical BCG immunotherapy in NMIBC with respect to reducing disease recurrence, preventing disease progression, and improving disease-specific survival is now well documented and generally accepted by most scientists and clinicians. In spite of this, much controversy still exists regarding how best to implement its use in clinical practice. Maintenance therapy is crucial for improving outcomes, but the optimal instillation protocols are still a matter of debate. Through the measurement of various urinary immune cytokines and inflammatory cells, there is a mounting body of evidence to suggest that maintenance instillation regimes might be tailored to the individual patient in order to produce the desired outcome.

The concept of maintenance therapy for all has its limitations, insofar as there is a substantial dropout rate in the $1 \mathrm{st}$ year of treatment, so not all patients receive the recommended regime anyway. Moreover, even with the generally accepted optimal treatment schemes, around 30\% will still develop recurrence. Despite the high-level evidence in support of maintenance therapy, there is arguably a subset of patients who are ultimately overtreated, and given the costs, inconvenience, and side effects associated with BCG (with a lack of evidence in support of reduced maintenance dose protocols), pursuit of alternative methods seems justified.

Recent research also suggests that there may be a difference between the various strains of $\mathrm{BCG}$, although this is controversial and requires validation in larger-scale studies. The significance of this finding might be the identification of particular genes present in the more effective strains of BCG, which could provide novel targets for drug development.

A key target of future research should be to identify why certain patients fail to respond to BCG in the manner hoped for and to establish whether immunotherapy can be 
manipulated to benefit this subset of patients. This potentially might be achieved through augmentation of BCG with additional cytokines added to the instillation solution. Augmentation of BCG might also be achieved through development of recombinant $\mathrm{BCG}$, which would be engineered to secrete its own array of proinflammatory antitumor agents. This concept is gaining considerable interest, but further research and trials are required to establish its safety and clinical efficacy. Combination with PDT may also have a role to play, either when BCG is initiated or perhaps as a second-line therapy in patients who have failed BCG, particularly where cystectomy is not a safe or feasible option.

As the understanding of the immunological response to BCG in the urothelium and tumor cells develops, several candidates are being identified which may support the development of future targets for immune-based therapies. These include utilization of various ILs, IFN, TLRs, and TRAIL. One benefit of this approach is to avoid the potentially serious effects of local and systemic BCG infection. This review has briefly discussed the potential use of $\mathrm{BCG}-\mathrm{CW}$ via nanoparticle delivery techniques, and although promising, it remains to be seen whether this technique will achieve effective clinical outcomes. Novel techniques involving the implementation of gene and virus therapy may also have a role to play in the future treatment of NMIBC, but again these ideas are very much in the embryonic stages at present.

The potential role of monoclonal antibodies in the treatment of bladder cancer has been highlighted by recent research involving manipulation of the immune checkpoint system in metastatic bladder cancer. This has shown promise, but its potential role in BCG-refractory superficial disease remains unclear. Further experimental and clinical research is required to test these theories and techniques, and it is hoped that they may result in effective therapies in the not too distant future.

\section{Disclosure}

The authors report no conflicts of interest in this work.

\section{References}

1. Morales A, Eidinger D, Bruce AW. Intracavitary Bacillus CalmetteGuerin in the treatment of superficial bladder tumors. J Urol. 1976;116(2): 180-183.

2. Joudi FN, Smith BJ, O’Donnell MA; National BCG-Interferon Phase 2 Investigator Group. Final results from a national multicenter phase II trial of combination bacillus Calmette-Guérin plus interferon $\alpha-2 B$ for reducing recurrence of superficial bladder cancer. Urol Oncol. 2006;24(4): 344-348.

3. Siegel R, Naishadham D, Jemal A. Cancer statistics, 2012. CA Cancer J Clin. 2012;62(1):10-29.
4. Sylvester RJ, van der Meijden AP, Lamm DL. Intravesical bacillus Calmette-Guerin reduces the risk of progression in patients with superficial bladder cancer: a meta-analysis of the published results of randomized clinical trials. J Urol. 2002;168(5):1964-1970.

5. Witjes J, Palou J, Soloway M, et al. Current clinical practice gaps in the treatment of intermediate- and high-risk non-muscle invasive bladder cancer (NMIBC) with emphasis on the use of bacillus Calmette- Guérin (BCG): results of an international individual patient data survey (IPDS). BJU Int. 2013;112(6):742-750.

6. Patschan $\mathrm{O}$, Holmäng $\mathrm{S}$, Hosseini $\mathrm{A}$, et al. Use of bacillus CalmetteGuérin in stage T1 bladder cancer: long-term observation of a population-based cohort. Scand J Urol. October 21, 2014. [Epub ahead of print.]

7. Venkataswamy MM, Goldberg MF, Baena A, Chan J, Jacobs WR Jr, Porcelli SA. In vitro culture medium influences the vaccine efficacy of Mycobacterium bovis BCG. Vaccine. 2012;30(6):1038-1049.

8. Oettinger T, Jorgensen M, Ladefoged A, Haslov K, Andersen P. Development of the Mycobacterium bovis BCG vaccine: review of the historical and biochemical evidence for a genealogical tree. Tuber Lung Dis. 1999;79(4):243-250.

9. Colditz GA, Brewer TF, Berkey CS, et al. Efficacy of BCG vaccine in the prevention of tuberculosis. Meta-analysis of the published literature. JAMA. 1994;271(9):698-702.

10. Fine PE. Variation in protection by BCG: implications of and for heterologous immunity. Lancet. 1995;346(8986):1339-1345.

11. Pearl R. Cancer and tuberculosis. Am J Hygiene. 1929;9:97-159.

12. Redelman-Sidi G, Glickman MS, Bochner BH. The mechanism of action of BCG therapy for bladder cancer. Nat Rev Urol. 2014;11(3): $153-162$.

13. Gandhi N, Morales A, Lamm D. Bacillus Calmette-Guerin immunotherapy for genitourinary cancer. BJU Int. 2013;112(3):288-297.

14. Alexandroff AB, Jackson AM, O'Donnell MA, James K. BCG immunotherapy of bladder cancer: 20 years on. Lancet. 1999;353(9165): 1689-1694.

15. Kawai K, Miyazaki J, Joraku A, Nishiyama H, Akaza H. Bacillus Calmette-Guerin (BCG) immunotherapy for bladder cancer: current understanding and perspectives on engineered BCG vaccine. Cancer Sci. 2013;104(1):22-27.

16. Ratliff TL, Kavoussi LR, Catalona WJ. Role of fibronectin in intravesical BCG therapy for superficial bladder cancer. J Urol. 1988;139(2): 410-414.

17. Kavoussi LR, Brown EJ, Ritchey JK, RatliffTL. Fibronectin-mediated Calmette-Guerin bacillus attachment to murine bladder mucosa. Requirement for the expression of an antitumor response. J Clin Invest. 1990;85(1):62-67.

18. Saban MR, Simpson C, Davis C, et al. Discriminators of mouse bladder response to intravesical Bacillus Calmette-Guerin (BCG). BMC Immunol. 2007;8:6.

19. Kuroda K, Brown EJ, Telle WB, Russell DG, RatliffTL. Characterization of the internalization of bacillus Calmette-Guerin by human bladder tumor cells. J Clin Invest. 1993;91(1):69-76.

20. Bevers RF, de Boer EC, Kurth KH, Schamhart DH. BCG-induced interleukin-6 upregulation and BCG internalization in well and poorly differentiated human bladder cancer cell lines. Eur Cytokine Netw. 1998;9(2):181-186.

21. Ratliff T, Ritchey J, Yuan J, Andriole G, Catalona W. T-cell subsets required for intravesical BCG immunotherapy for bladder cancer. J Urol. 1993;150(3):1018-1023.

22. Maksymowych WP, Kane KP. Bacterial modulation of antigen processing and presentation. Microbes Infect. 2000;2(2):199-211.

23. Ikeda N, Toida I, Iwasaki A, Kawai K, Akaza H. Surface antigen expression on bladder tumor cells induced by bacillus Calmette-Guerin (BCG): a role of BCG internalization into tumor cells. Int J Urol. 2002;9(1): $29-35$.

24. Lattime EC, Gomella LG, McCue PA. Murine bladder carcinoma cells present antigen to BCG-specific CD4+ T-cells. Cancer Res. 1992;52(15):4286-4290. 
25. Jackson A, Alexandroff A, Kelly R. Changes in urinary cytokines and soluble intercellular adhesion molecule-1 (ICAM-1) in bladder cancer patients after Bacillus Calmette-Guerin (BCG) immunotherapy. Clin Exp Immunol. 1995;99(3):369-375.

26. De Boer EC, De Jong WH, Steerenberg PA, et al. Induction of urinary interleukin-1 (IL-1), IL-2, IL-6, and tumour necrosis factor during intravesical immunotherapy with bacillus Calmette-Guerin in superficial bladder cancer. Cancer Immunol Immunother. 1992;34(5):306-312.

27. De Boer EC, Somogyi L, de Ruiter GJ, de Reijke TM, Kurth KH, Schamhart DH. Role of interleukin- 8 in onset of the immune response in intravesical BCG therapy for superficial bladder cancer. Urol Res. 1997;25(1):31-34.

28. Prescott S, James K, Hargreave TB, Chisholm GD, Smyth JF. Intravesical Evans strain BCG therapy: quantitative immunohistochemical analysis of the immune response within the bladder wall. $J$ Urol. 1992;147(6):1636-1642.

29. Prescott S, James K, Hargreave TB, Chisholm GD, Smyth JF. Radioimmunoassay detection of interferon-gamma in urine after intravesical Evans BCG therapy. J Urol. 1990;144(5):1248-1251.

30. Takeuchi A, Dejima T, Yamada H, et al. IL-17 production by CD T cells is important for the antitumor effect of Mycobacterium bovis bacillus Calmette-Guerin treatment against bladder cancer. Eur J Immunol. 2011;41(1):246-251.

31. Patard J, Muscatelli-Groux B, Saint F, et al. Evaluation of local immune response after intravesical bacillus Calmette-Guerin treatment for superficial bladder cancer. Br J Urol. 1996;78(5):709-714.

32. Brandau S, Riemensberger J, Jacobsen M, et al. NK cells are essential for effective BCG immunotherapy. Int J Cancer. 2001;92(5):697-702.

33. Simons M, O’Donnell M, Griffith T. Role of neutrophils in BCG immunotherapy for bladder cancer. Urol Oncol. 2008;26(4):341-345.

34. Saint F, Patard JJ, Irani J, et al. Leukocyturia as a predictor of tolerance and efficacy of intravesical BCG maintenance therapy for superficial bladder cancer. Urology. 2001;57(4):617-621.

35. Luo Y, Knudson MJ. Mycobacterium bovis bacillus Calmette-Guerininduced macrophage cytotoxicity against bladder cancer cells. Clin Dev Immunol. 2010;2010:357591.

36. Naoe M, Ogawa Y, Takeshita K, et al. Bacillus Calmette-Guerin-pulsed dendritic cells stimulate natural killer T cells and gamma delta T cells. Int J Urol. 2007;14(6):532-538.

37. Thiounn N, Pages F, Mejean A, Descotes JL, Fridman WH, RometLemonne JL. Adoptive immunotherapy for superficial bladder cancer with autologous macrophage activated killer cells. J Urol. 2002;168(6): 2373-2376.

38. Ayari $\mathrm{C}$, LaRue $\mathrm{H}$, Hovington $\mathrm{H}$, et al. Bladder tumor infiltrating mature dendritic cells and macrophages as predictors of response to bacillus Calmette-Guérin immunotherapy. Eur Urol. 2009;55(6):1386-1395.

39. Ludwig AT, Moore JM, Luo Y, et al. Tumor necrosis factor-related apoptosis-inducing ligand: a novel mechanism for Bacillus CalmetteGuérin-induced antitumor activity. Cancer Res. 2004;64(10): 3386-3390.

40. Babjuk M, Burger M, Zigeuner R, et al; European Association of Urology. EAU guidelines on non-muscle-invasive urothelial carcinoma of the bladder: update 2013. Eur Urol. 2013;64(4):639-653.

41. Duchek M, Johansson R, Jahnson S, et al; Members of the Urothelial Cancer Group of the Nordic Association of Urology. Bacillus CalmetteGuérin is superior to a combination of epirubicin and interferon-a2b in the intravesical treatment of patients with stage $\mathrm{T} 1$ urinary bladder cancer. A prospective, randomized, Nordic study. Eur Urol. 2010;57(1):25-31.

42. Järvinen R, Kaasinen E, Sankila A, Rintala E. Long-term efficacy of maintenance bacillus Calmette-Guérin versus maintenance mitomycin $\mathrm{C}$ instillation therapy in frequently recurrent TaT1 tumours without carcinoma in situ: a subgroup analysis of the prospective, randomised FinnBladder I study with a 20-year follow-up. Eur Urol. 2009;56(2): 260-265.

43. Shang PF, Kwong J, Wang ZP, et al. Intravesical Bacillus CalmetteGuérin versus epirubicin for Ta and T1 bladder cancer. Cochrane Database Syst Rev. 2011;11(5):CD006885.
44. Morales A. Treatment of carcinoma in situ of the bladder with BCG: a phase II trial. Cancer Immunol Immunother. 1980;9(1-2):69-72.

45. Lamm DL, Blumenstein BA, Crissman JD, et al. Maintenance bacillus Calmette-Guerin immunotherapy for recurrent TA, T1 and carcinoma in situ transitional cell carcinoma of the bladder: a randomized Southwest Oncology Group Study. J Urol. 2000;163(4):1124-1129.

46. Böhle A, Bock PR. Intravesical bacillus Calmette-Guerin versus mitomycin $\mathrm{C}$ in superficial bladder cancer: formal meta-analysis of comparative studies on tumour progression. Urology. 2004;63(4): 682-686.

47. Sylvester RJ, Brausi MA, Kirkels WJ, et al. Long-term efficacy results of EORTC genito-urinary group randomized phase 3 study 30911 comparing intravesical instillations of epirubicin, bacillus CalmetteGuérin, and bacillus Calmette-Guérin plus isoniazid in patients with intermediate- and high-risk stage Ta T1 urothelial carcinoma of the bladder. Eur Urol. 2010;57(5):766-773

48. Herr HW, Morales A. History of bacillus Calmette-Guerin and bladder cancer: an immunotherapy success story. J Urol. 2008;179(1): 53-56.

49. Lamm DL, Thor DE, Harris SC, Reyna JA, Stogdill VD, Radwin HM. Bacillus Calmette-Guerin immunotherapy of superficial bladder cancer. J Urol. 1980;124(1):38-40.

50. Pinsky CM, Camacho FJ, Kerr D, et al. Intravesical administration of bacillus Calmette-Guérin in patients with recurrent superficial carcinoma of the urinary bladder: report of a prospective, randomized trial. Cancer Treat Rep. 1985;69(1):47-53.

51. Herr HW. Extravesical tumor relapse in patients with superficial bladder tumors. J Clin Oncol. 1998;16(3):1099-1102.

52. Zlotta AR, van Vooren JP, Huygen $\mathrm{K}$, et al. What is the optimal regimen for BCG intravesical therapy? Are six weekly instillations necessary? Eur Urol. 2000;37(4):470-477.

53. Oddens J, Brausi M, Sylvester R, et al. Final results of an EORTC-GU Cancers Group randomized study of maintenance bacillus CalmetteGuérin in intermediate- and high-risk Ta, T1 papillary carcinoma of the urinary bladder: one-third dose versus full dose and 1 year versus 3 years of maintenance. Eur Urol. 2013;63(3):462-472.

54. Palou J, Laguna P, Millan-Rodriguez F, Hall RR, Salvador-Bayarri J, Vicente-Rodriguez J. Control group and maintenance treatment with bacillus Calmette-Guerin for carcinoma in situ and/or high grade bladder tumors. J Urol. 2001;165(5):1488-1491.

55. Brausi M, Oddens, Sylvester R, et al. Side effects of Bacillus CalmetteGuerin (BCG) in the treatment of intermediate- and high-risk Ta, T1 papillary carcinoma of the bladder: results of the EORTC genito-urinary cancers group randomised phase 3 study comparing one-third dose with full dose and 1 with 3 years maintenance BCG. Eur Urol. 2014;65(1): $69-76$.

56. Herr H, Dalbagni G, Donat S. Bacillus Calmette-Guerin without maintenance therapy for high-risk non-muscle-invasive bladder cancer. Eur Urol. 2011;60(1):32-36.

57. Pfister C, Kerkeni W, Rigaud J, et al; Cancer Committee of the French Urological Association. Efficacy and tolerance of one-third full dose bacillus Calmette-Guérin maintenance therapy every 3 months or 6 months: two-year results of URO-BCG-4 multicenter study. Int $J$ Urol. 2015;22(1):53-60.

58. Ojea A, Nogueira J, Solsona E, et al; CUETO Group (Club Urológico Español De Tratamiento Oncológico). A multicentre, randomised prospective trial comparing three intravesical adjuvant therapies for intermediate-risk superficial bladder cancer: low-dose bacillus CalmetteGuerin $(27 \mathrm{mg}$ ) versus very low-dose bacillus Calmette-Guerin (13.5 mg) versus mitomycin C. Eur Urol. 2007;52(5):1398-1406.

59. Martinez-Pineiro J, Flores N, Isorna S. Long-term follow up of randomised prospective trial comparing a standard $81 \mathrm{mg}$ dose of intravesical bacillus Calmette-Guerin with a reduced dose of $27 \mathrm{mg}$ in superficial bladder cancer. BJU Int. 2002;89(7):671-680.

60. Lamm DL. Efficacy and safety of bacille Calmette-Guérin immunotherapy in superficial bladder cancer. Clin Infect Dis. 2000;31 Suppl 3: S86-S90. 
61. Lüftenegger W, Ackermann DK, Futterlieb A, et al. Intravesical versus intravesical plus intradermal bacillus Calmette-Guerin: a prospective randomized study in patients with recurrent superficial bladder tumors. J Urol. 1996;155(2):483-487.

62. Van der Meijden A, Brausi M, Zambon V, Kirkels W, de Balincourt C, Sylvester R. Intravesical instillation of epirubicin, bacillus CalmetteGuérin and bacillus Calmette-Guérin plus isoniazid for intermediate and high risk Ta, T1 urothelial carcinoma of the bladder: a European Organization for Research and Treatment of Cancer Genito-Urinary Group randomized phase III trial. J Urol. 2001;166(2): 476-481.

63. Lamm DL, van der Meijden APM, Morales A, et al. Incidence and treatment of complications of bacillus Calmette-Guérin intravesical therapy in superficial bladder cancer. $J$ Urol. 1992;147(3): 596-600.

64. Yossepowitch O, Eggener S, Bochner B. Safety and efficacy of intravesical bacillus Calmette-Guerin instillations in steroid treated and immunocompromised patients. J Urol. 2006;176(2):482-485.

65. Palou J, Angerri O, Segarra J, et al. Intravesical bacillus CalmetteGuerin for the treatment of superficial bladder cancer. Transplantation. 2003;76(10):1514-1516.

66. Colombel M, Saint F, Chopin D, Nicolas L, Rischmann P. The effect of ofloxacin on Bacillus Calmette-Guerin induced toxicity in patients with superficial bladder cancer: results of a randomized, prospective, doubleblind, placebo controlled, multicenter study. J Urol. 2006;176(3): 935-939.

67. Vegt PD, van der Meijden AP, Sylvester R, Brausi M, Höltl W, de Balincourt C. EORTC Genito-Urinary Group: does isoniazid reduce side-effects of intravesical Bacillus Calmette-Guerin therapy in superficial bladder cancer? Interim results of cancer protocol 30911. J Urol. 1997;157(4):1246-1249.

68. Wijes J, Palou J, Soloway M, et al. Clinical practice recommendations for the prevention and management of intravesical therapy-associated adverse events. Eur Urol Suppl. 2008;7(10):667-674.

69. Rentsch CA, Birkhäuser FD, Biot C, et al. Bacillus Calmette-Guérin strain differences have an impact on clinical outcome in bladder cancer immunotherapy. Eur Urol. 2014;66(4):677-688.

70. Nakamura T, Fukiage M, Higuchi M, et al. Nanoparticulation of BCGCWS for application to bladder cancer therapy. $J$ Control Release. 2014;176:44-53.

71. Joraku A, Homhuan A, Kawai K, et al. Immunoprotection against murine bladder carcinoma by octaarginine-modified liposomes incorporating cell wall of Mycobacterium bovis bacillus Calmette-Guerin. BJU Int. 2009;103(5):686-693.

72. Kogure K, Moriguchi R, Sasaki K, Ueno M, Futaki S, Harashima H. Development of a non-viral multifunctional envelope-type nano device by a novel lipid film hydration method. J Control Release. 2004;98(2): 317-323.

73. LaRue H, Ayari C, Bergeron A, Fradet Y. Toll-like receptors in urothelial cells - targets for cancer immunotherapy. Nat Rev Urol. 2013;10(9): $537-545$.

74. Falke J, Lammers R, Arentsen H, et al. Results of a phase 1 dose escalation study of intravesical TMX-101 in patients with nonmuscle invasive bladder cancer. J Urol. 2013;189(6):2077-2082.

75. Morales A, Phadke K, Steinhoff G. Intravesical mycobacterial cell wall-DNA complex in the treatment of carcinoma in situ of the bladder after standard intravesical therapy has failed. J Urol. 2009;181(3): 1040-1045.

76. Luo Y, Henning, O’Donnell M. Th1 cytokine-secreting recombinant Mycobacterium bovis Bacillus Calmette-Guerin and prospective use in immunotherapy of bladder cancer. Clin Dev Immunol. 2011;2011:728930.

77. Burke J. Virus therapy for bladder cancer. Cytokine Growth Factor Rev. 2010;21(2-3):99-102.
78. Gomella LG, Mastrangelo MJ, McCue PA, Maguire HC Jr, Mulholland SG, Lattime EC. Phase 1 study of intravesical vaccinia virus as a vector for gene therapy of bladder cancer. $J$ Urol. 2001;166(4): 1291-1295.

79. Pagliaro LC, Keyhani A, Williams D, et al. Repeated intravesical instillations of an adenoviral vector in patients with locally advanced bladder cancer: a phase I study of p53 gene therapy. J Clin Oncol. 2003;21(12): 2247-2253.

80. Burke JM, Lamm DL, Meng MV, et al. A first in human phase 1 study of CG0070, a GM-CSF expressing oncolytic adenovirus, for the treatment of nonmuscle invasive bladder cancer. J Urol. 2012;188(6): 2391-2397.

81. Pinthus JH, Bogaards A, Weersink R, Wilson BC, Trachtenberg J. Photodynamic therapy for urological malignancies: past to current approaches. J Urol. 2006;175(4):1201-1207.

82. Castano A, Mroz P, Hamblin M. Photodynamic therapy and anti-tumour immunity. Nat Rev Cancer. 2006;6(7):535-545.

83. Uchibayashi T, Koshida K, Kunimi K, Hisazumi H. Whole bladder wall photodynamic therapy for refractory carcinoma in situ of the bladder. Br J Cancer. 1995;71(3):625-628.

84. Dugan M, Crawford E, Nseyo U. Photodynamic therapy (PDT) after transurethral resection (TUR) for superficial papillary bladder carcinoma (SBC): a randomized trial. Proc ASCO. 1991;10:173.

85. Nseyo U, Dehaven J, Dougherty T, et al. Photodynamic therapy (PDT) in the management of patients with resistant superficial bladder cancer: a long term experience. J Clin Laser Med Surg. 1998;16(1):61-68.

86. Berger A, Steniner H, Stenzi A, Akkad T, Bartsch G, Holtl L. Photodynamic therapy with intravesical instillation of 5-aminolevulinic acid for patients with recurrent superficial bladder cancer: a single-center study. Urology. 2003;61(2):338-341.

87. Kriegmair M, Baumgartner R, Lumper W, Waidelich R, Hofstetter A. Early clinical experience with 5-aminolevulinic acid for the photodynamic therapy of superficial bladder cancer. Br J Urol. 1996;77(5): 667-671.

88. Lee JY, Diaz RR, Cho KS, et al. Efficacy and safety of photodynamic therapy for recurrent, high grade nonmuscle invasive bladder cancer refractory or intolerant to bacille Calmette-Guérin immunotherapy. J Urol. 2013;190(4):1192-1199.

89. Bader MJ, Stepp H, Beyer W, et al. Photodynamic therapy of bladder cancer - a phase I study using hexaminolevulinate (HAL). Urol Oncol. 2013;31(7):1178-1183.

90. Bozzini G, Colin P, Betrouni N, et al. Photodynamic therapy in urology: what can we do now and where are we heading? Photodiagnosis Photodyn Ther. 2012;9(3):261-273.

91. Korbelik M, Cecic I. Enhancement of tumour response to photodynamic therapy by adjuvant mycobacterium cell-wall treatment. $J$ Photochem Photobiol B. 1998;44(2):151-158.

92. Korbelik M, Sun J, Posakony JJ. Interaction between photodynamic therapy and BCG immunotherapy responsible for the reduced recurrence of treated mouse tumors. Photochem Photobiol. 2001;73(4):403-409.

93. Huang Z. A review of progress in clinical photodynamic therapy. Cancer Res Treat. 2005;4(3):283-293.

94. Nakanishi J, Wada Y, Matsumoto K, Azuma M, Kikuchi K, Ueda S. Overexpression of B7-H1 (PD-L1) significantly associates with tumor grade and postoperative prognosis in human urothelial cancers. Cancer Immunol Immunother. 2007;56(8):1173-1182.

95. Powles T, Eder JP, Fine G, et al. MPDL3280A (anti-PD-L1) treatment leads to clinical activity in metastatic bladder cancer. Nature. 2015; 515(7528):558-562.

96. Newton M, Askeland E, Andresen E, et al. Anti-interleukin-10R1 monoclonal antibody in combination with bacillus Calmette-Guérin is protective against bladder cancer metastasis in a murine orthotopic tumour model and demonstrates systemic specific anti-tumour immunity. Clin Exp Immunol. 2014;177(1):261-268. 
Research and Reports in Urology

\section{Publish your work in this journal}

Research and Reports in Urology is an international, peer-reviewed, open access journal publishing original research, reports, editorials, reviews and commentaries on all aspects of adult and pediatric urology in the clinic and laboratory including the following topics: Pathology, pathophysiology of urological disease; Investigation and treatment of

urological disease; Pharmacology of drugs used for the treatment of urological disease. The manuscript management system is completely online and includes a very quick and fair peer-review system, which is all easy to use. Visit http://www.dovepress.com/testimonials.php to read real quotes from published authors.

Submit your manuscript here: http://www.dovepress.com/research-and-reports-in-urology-journal 